\title{
Performing High-resolution Impedance Manometry After Endoscopy With Conscious Sedation Has Negligible Effects on Esophageal Motility Results
}

\author{
Hui Su, ${ }^{1,2}$ Dustin A Carlson, ${ }^{2 *}$ Erica Donnan, ${ }^{2}$ Wenjun Kou, ${ }^{2}$ Jacqueline Prescott, ${ }^{2}$ Alex Decorrevont, ${ }^{2}$ Francesca Shilati, ${ }^{2}$ \\ Melina Masihi, ${ }^{2}$ and John E Pandolfino ${ }^{2}$ \\ ${ }^{\prime}$ Department of Gastroenterology, Capital Medical University Affiliated Beijing Shijitan Hospital, Beijing, China; and ${ }^{2}$ Division of \\ Gastroenterology and Hepatology, Department of Medicine, Feinberg School of Medicine, Northwestern University, Chicago, IL, USA
}

\section{Background/Aims}

High-resolution manometry (HRM) performed without sedation is the standard procedure. However, some patients cannot tolerate transnasal placement of the manometry catheter. We aim to assess the practice of performing manometry after endoscopy with conscious sedation by evaluating its impact on esophageal motility findings.

\section{Methods}

Twelve asymptomatic adult volunteers and 7 adult patients completed high-resolution impedance manometry (HRIM) approximately 1 hour after conscious sedation with midazolam and fentanyl (post-sedation) and again on a different day with no-sedation. The nosedation HRIM involved 2 series of swallows separated in time by 20 minutes (no-sedation-1 and no-sedation-2) for the volunteers; patients completed only 1 series of swallows for no-sedation HRM.

\section{Results}

A motility diagnosis of normal motility was observed in all 12 volunteers post-sedation. Two volunteers had a diagnosis of borderline ineffective esophageal motility, one during the no-sedation-1 period and the other during the no-sedation-2 period; all of the other no-sedation HRIM studies yielded a normal motility diagnosis. Six of seven patients had the same diagnosis in both no-sedation and post-sedation HRM, including 1 distal esophageal spasm, 3 achalasia (2 type II and 1 type III), and 2 esophagogastric junction outflow obstruction. Only one patient's HRM classification changed from ineffective esophageal motility at no-sedation to normal esophageal motility at post-sedation.

\section{Conclusions}

Performing HRIM after endoscopy with conscious sedation had minimal clinical impact on the motility diagnosis or motility parameters. Thus, this approach may be a viable alternative for patients who cannot tolerate unsedated catheter placement.

\section{(J Neurogastroenterol Motil 2020;26:352-361)}

\section{Key Words}

Esophageal achalasia; Fentanyl; Midazolam; Swallows; Volunteers

\section{Received: January 14, 2020 Revised: None Accepted: March 29, 2020}

(a) This is an Open Access article distributed under the terms of the Creative Commons Attribution Non-Commercial License (http://creativecommons. org/licenses/by-nc/4.0) which permits unrestricted non-commercial use, distribution, and reproduction in any medium, provided the original work is properly cited.

*Correspondence: Dustin A Carlson, MD, MS

Division of Gastroenterology and Hepatology, Department of Medicine, Feinberg School of Medicine, Northwestern University, 676 St Clair St, Suite 1400, Chicago, IL 60611-2951, USA

Tel: +1-312-926-4939, Fax: +1-312-695-3999, E-mail: dustin-carlson@northwestern.edu 


\section{Introduction}

Esophageal manometry is widely used in clinical practice for the assessment of esophageal motility in patients with dysphagia, chest pain, and gastroesophageal reflux disease who require placement of a $\mathrm{pH}$ catheter and a pre-operative evaluation for antireflux surgery. The procedure is usually performed without sedation in an outpatient setting. However, the transnasal catheter placement required for the manometric procedure is unpleasant and uncomfortable for many patients, even with use of topical anesthesia of the nares and/or throat. Unfortunately, some patients are not able to tolerate the manometric procedure at all, thus preventing the completion of the exam.

Conscious sedation facilitates performance of endoscopy and improves the patient's tolerance and compliance for gastrointestinal examination. ${ }^{1}$ Additionally, previous studies have reported sedationassisted esophageal manometry, which may aid completion of manometry for patients who could not tolerate the test during awake placement of the manometry catheter. ${ }^{2-4}$ Placing the catheter while the patient is under sedation can help mitigate some of the discomfort and help complete the exam. Additionally, some situations require endoscopic-guidance of the manometric catheter to achieve the necessary positioning across the esophagogastric junction, eg, esophageal tortuosity with large hiatal hernia or achalasia. The manometry study can then be performed after the sedation effects clear when the patient finishes recovery.

However it is unclear whether conscious sedation has an impact on the result of the subsequent esophageal manometry. Multiple studies support that manometric findings can be altered when patients are studied on opioids, which are associated with major motility diagnoses, such as type III achalasia, esophagogastric junction outflow obstruction (EGJOO), and Jackhammer esophagus. ${ }^{5-8}$ Studies performed assessing the effects of intravenous midazolam are conflicting in regards to the impact on lower esophageal pressure. ${ }^{9,10}$ Another consequence of the practice of placing the manometry catheter while the patient is under sedation is the prolonged period of time the catheter remains within the esophagus. This raises the potential for thermal-pressure drift when utilizing solid-state high-resolution manometry (HRM) assemblies, which may also have an impact on the results of the esophageal manometry test. ${ }^{11}$

Given these issues, we aim to assess whether performance of manometry after sedated endoscopic placement could alter esophageal motility findings by performing a crossover study of asymptomatic volunteers and patients.

\section{Materials and Methods}

\section{Subjects}

Twelve healthy, asymptomatic (free of esophageal symptoms including dysphagia, heartburn, and chest pain), adult volunteers were enrolled in our study. All of them signed the written informed consent. The study protocol was approved by the Northwestern University Institutional Review Board (IRB No. NU IRB STU00096856).

The volunteer group have been previously described. ${ }^{12}$ Exclusion criteria for the volunteer group included: previous diagnosis of esophageal, autoimmune, history of upper gastrointestinal surgery, eating disorders, use of antacids or proton pump inhibitors, or a history of tobacco use or alcohol abuse, and long-term use of opioids drug.

Additionally, our esophageal center motility laboratory registry was retrospectively reviewed to identify patients that completed HRM without sedation (no-sedation) at an external hospital, and then completed HRM after placement of the HRM catheter during sedated endoscopy at our center (ie, post-sedation). Patients with previous foregut surgery or with endoscopic or surgical treatment between the $2 \mathrm{HRM}$ tests were excluded. All of the patients signed the written informed consent.

\section{Study Protocol}

Each volunteer participated in the study and completed the high-resolution impedance manometry (HRIM) on 2 separate study days. The study focused on assessing the difference between post-sedation and unsedated placement was conceptualized after we had obtained volunteer data using the post-sedation protocol. Thus, the time between the "post-sedation" study and the "no-sedation" study was approximately 1 year on average (mean 353 days [SD 176 days]).

On the first study day ("post-sedation"), evaluation was completed after a minimum 6-hour fast. Healthy volunteer subjects first underwent upper endoscopy in the left lateral decubitus position. Conscious sedation with 5-10 mg midazolam and 100-200 $\mu \mathrm{g}$ fentanyl was administered during the endoscopic procedure. Immediately after endoscopic examination, the HRIM assembly (4.2-mm outer diameter solid-state assembly with 36 circumferential pressure sensors at $1-\mathrm{cm}$ intervals and 18 impedance segments at 2-cm intervals; Medtronic Inc, Shoreview, MN, USA) was placed transnasally and ultimately positioned to record from the hypopharynx 
to the stomach with approximately 3 intragastric pressure sensors. The HRIM protocol consisted of a 2-minute baseline recording, ten $5-\mathrm{mL}$ liquid swallows in a supine position and five $5-\mathrm{mL}$ liquid swallows in the upright position was performed after a time period for recovery from sedation. The recovery period was approximately 1 hour to a point when the subjects could cooperate with the manometry protocol; the exact time difference from last sedation dosing to the start time of the HRIM test was not measured.

On the second study day ("no-sedation"), healthy volunteer subjects completed the manometry protocol without any preceding sedation. After a minimum 6-hour fast, the HRIM catheter was placed transnasally and positioned to record from the hypopharynx to the stomach with approximately 3 intragastric pressure sensors. The HRIM protocol consisted of a 2-minute baseline recording, ten $5-\mathrm{mL}$ liquid swallows in a supine position and five 5-mL liquid swallows in the upright position ("no-sedation-1"). To assess for possible effects of thermal-pressure drift, a second series of 2-minute baseline recording, 10 supine swallows, and 5 upright swallows was performed after 20 minutes ("no-sedation-2") for the volunteer group.

For patients, all the patients completed HRM tests at an external hospital without use of sedation (no-sedation-1), and when referred to our center, completed HRM test after endoscopy with sedation in a similar manner as described with the volunteers (postsedation).

\section{Data Analysis}

Manometry studies were analyzed by ManoView version 3.0 analysis software (Medtronic) to measure basal manometry metrics, including basal esophageal gastric junction pressure (EGJP), integrated relaxation pressure (IRP), distal contractile integral (DCI), and distal latency (DL), the EGJP were measured at end-expiration during the baseline recording. Each swallow was designated as swallow types of normal, weak, failed, fragment, or hypercontractile, and an esophageal motility diagnosis was derived according to the Chicago classification version $3.0\left(\mathrm{CC}\right.$ v3.0). ${ }^{13}$

The HRIM data of volunteer group for each subject were also exported to text file for each swallow. Per-swallow data were then analyzed using a customized python program to output bolus flow time (BFT) and esophageal impedance integral ratio (EII-ratio). Note that the methodology to calculate BFT and EII-ratio are explained in detail in our previous publications. ${ }^{14,15}$

Among patients completing HRM outside of our institution, only 1 patient completed HRM that also included impedance and only 2 patients had upright swallows included in their HRM protocol. Thus, comparisons between no-sedation and post-sedation for the patient group did not include impedance metrics or upright parameters.

\section{Statistical Methods}

Data were consolidated from the study sites and entered into SPSS version 25.0 for analysis (IBM, Armonk, NY, USA). The median value of each HRIM parameter were utilized for each volunteer. Descriptive statistics for all continuous measures were presented as median and interquartile range (IQR), unless otherwise stated.

For the HRIM parameters with continuous output, intrasubject comparisons were made using repeat measure ANOVA or Friedmann's test according to distribution of data for volunteer group using paired $t$ test or Wilcoxon rank-sum test according to distribution of data for patient group. Statistical significance was accepted when $P<0.05$; a Bonferroni correction was applied to account for multiple tests.

\section{Results}

\section{Subjects}

Twelve healthy, asymptomatic, adult volunteers were enrolled in our study, 9 female, 3 male, and mean age was 31 (range, 23-44) years. The post-sedation and no-sedation testing days occurred at a mean 353 (SD, 176) days. Endoscopic examination was normal in all 12 volunteer subjects. Sedation dosages were an average of 9 (range, 7-10) mg midazolam and 193 (range, 15-200) $\mu \mathrm{g}$ fentanyl. One volunteer did not complete the second series of swallows during the no-sedation protocol due to intolerance.

Additionally, 7 patients, mean age 48 (range, 22-68) years, 1 female, and 6 male that completed no-sedation HRM externally and post-sedation HRM at our center were identified for retrospective analysis. The post-sedation and no-sedation testing days occurred at a mean 175 (range, 48-488) days. All of the patients did not complete the second series of swallows during the no-sedation visit as the studies were done at an outside hospital.

No early or late complications were recorded.

\section{The Impact on Esophageal Motility Diagnosis/Chicago Classification}

All 12 volunteers had a classification of normal motility at postsedation HRIM. One subject had a classification of ineffective esophageal motility (IEM) at no-sedation-1, who then had normal motility at no-sedation-2. Another subject had IEM at no-seda- 
tion-2, but had normal motility at no-sedation-1. The remainder of subjects had normal motility classified at no-sedation HRIM. Thus, although the esophageal motility classification changed in 2 subjects ( $16 \%$ of cohort) from normal motility at post-sedation to IEM at a no-sedation HRIM, both subjects also again had normal motility in one of 2 no-sedation HRIM supine swallow series.

Of the subjects with an observed change in $\mathrm{CC}$, the one subject had 10/10 supine swallows normal at post-sedation; IEM at nosedation-1 with 7/10 supine swallows failed and 3/10 normal swallows; and then normal motility at no-sedation-2 with 2/10 supine swallows failed and $8 / 10$ swallows normal. The other subject had 2/10 supine swallows failed, $1 / 10$ swallows weak, and 7/10 swallows normal at post-sedation; normal motility at no-sedation-1 with 2/10 supine swallows failed, $1 / 10$ swallows weak, and 7/10 supine swallows normal; and then IEM at no-sedation-2 with 8/10 supine swallows failed and 2/10 swallows weak. There was also one subject that had a single hypercontractile swallow (1/10 supine swallows; DCI $12918 \mathrm{mmHg} \cdot \mathrm{cm} \cdot \mathrm{sec})$ during the post-sedation and also
1/10 hypercontractile swallows (DCI $9366 \mathrm{mmHg} \cdot \mathrm{cm} \cdot \mathrm{sec}$ ) at nosedation-1; 10/10 swallows were normal at no-sedation-2.

Among the 7 patients, 6/7 patients had the same HRM diagnosis at both post-sedation and no-sedation HRM. HRM classifications included 1/7 distal esophageal spasm, 3/7 achalasia (2 type II and 1 type III), and 2/7 EGJOO. One patient had normal esophageal motility at post-sedation and IEM at no-sedation HRM. The normal motility classification at post-sedation involved 6/10 supine swallows normal, 2/10 supine swallows failed and 2/10 swallows weak while the IEM at no-sedation involved 7/10 supine swallows failed, 2/10 swallows weak, and 1/10 swallow premature.

\section{The Impact of Sedation on Standard High-resolution Manometry Metrics}

\section{The impact of sedation on standard high-resolution manometry metrics in supine in volunteer group (see Fig. 1 and Table)}

A

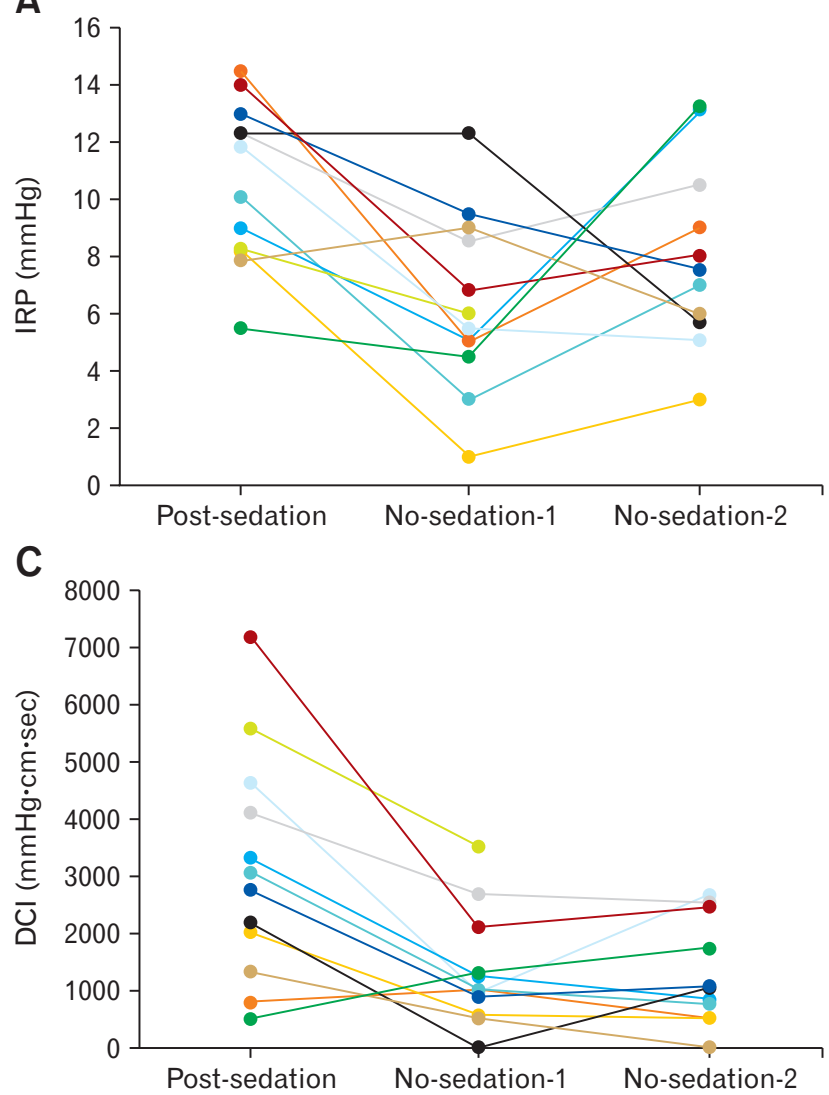

B

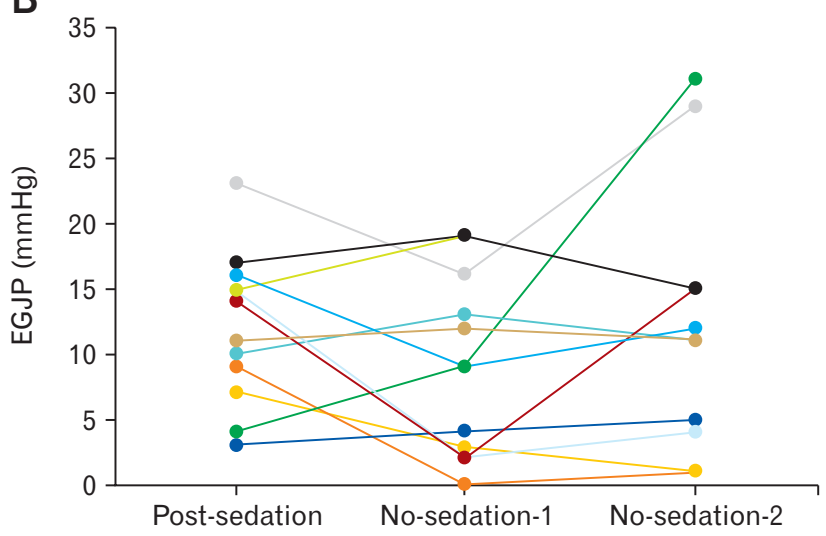

D

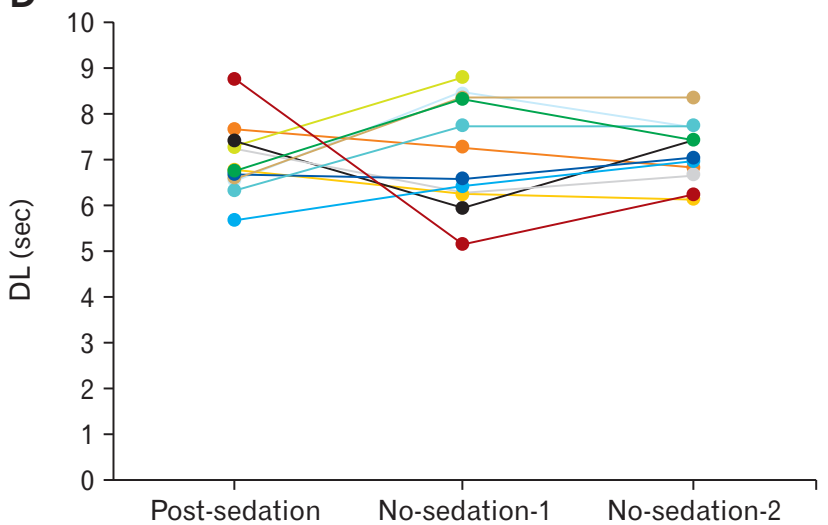

Figure 1. Comparison of supine high-resolution manometry (HRM) metrics of volunteer group. Median integrated relaxation pressure (IRP) (A), basal esophageal gastric junction pressure (EGJP) (B), distal contractile integral (DCI) (C), and distal latency (DL) (D) were compared across 3 test situations (post-sedation, no-sedation-1, and no-sedation-2) for all of 12 subjects. 
Table. The Impact of Sedation on High-resolution Impedance Manometry Metrics in Volunteer Group

\begin{tabular}{|c|c|c|c|c|}
\hline Metric & Post-sedation & No-sedation-1 & No-sedation-2 & $P$-value \\
\hline Supine IRP (mmHg) & $10.9(8.2,12.8)$ & $5.8(4.6,8.9)$ & $7.5(5.7,10.5)$ & 0.007 \\
\hline Supine EGJP (mmHg) & $12.5(7.5,15.8)$ & $9.0(2.3,15.3)$ & $11.0(4.0,15.0)$ & 0.528 \\
\hline Supine DCI $(\mathrm{mmHg} \cdot \mathrm{cm} \cdot \mathrm{sec})$ & $2920(1509,4497)$ & $1117(622,1897)$ & $1052(539,2461)$ & 0.004 \\
\hline Supine DL (sec) & $6.75(6.51,7.36)$ & $6.90(6.23,8.34)$ & $7.05(6.65,7.70)$ & 0.878 \\
\hline Upright IRP (mmHg) & $6.3(3.4,9.4)$ & $1.0(0.0,3.8)$ & $5.0(2.0,6.0)$ & 0.076 \\
\hline Upright EGJP (mmHg) & $8.0(4.3,18.5)$ & $7.0(0.8,20.00)$ & $11.0(1.0,17.0)$ & 0.359 \\
\hline Upright DCI (mmHg.cm·sec) & $2101(969,3020)$ & $791(227,1178)$ & $972(123,1010)$ & 0.009 \\
\hline Upright DL (sec) & $6.5(6.4,7.0)$ & $6.7(6.3,8.1)$ & $6.8(6.1,7.8)$ & 0.933 \\
\hline Supine BFT (sec) & $1.70(0.92,2.73)$ & $1.79(0.81,2.24)$ & $2.08(0.94,2.75)$ & 0.919 \\
\hline Supine EII ratio & $0.01(0.00,0.06)$ & $0.08(0.00,0.29)$ & $0.00(0.00,0.12)$ & 0.798 \\
\hline Upright BFT (sec) & $1.20(0.25,1.79)$ & $1.00(0.02,2.06)$ & $1.42(0.76,1.92)$ & 0.693 \\
\hline Upright EII ratio & $0.01(0.00,0.03)$ & $0.02(0.00,0.17)$ & $0.00(0.00,0.08)$ & 0.792 \\
\hline
\end{tabular}

IRP, integrated relaxation pressure; EGJP, esophageal gastric junction pressure; DCI, distal contractile integral; DL, and distal latency; BFT, bolus flow time; EIIratio, esophageal impedance integral ratio.

Data are presented as median (interquartile range).

A

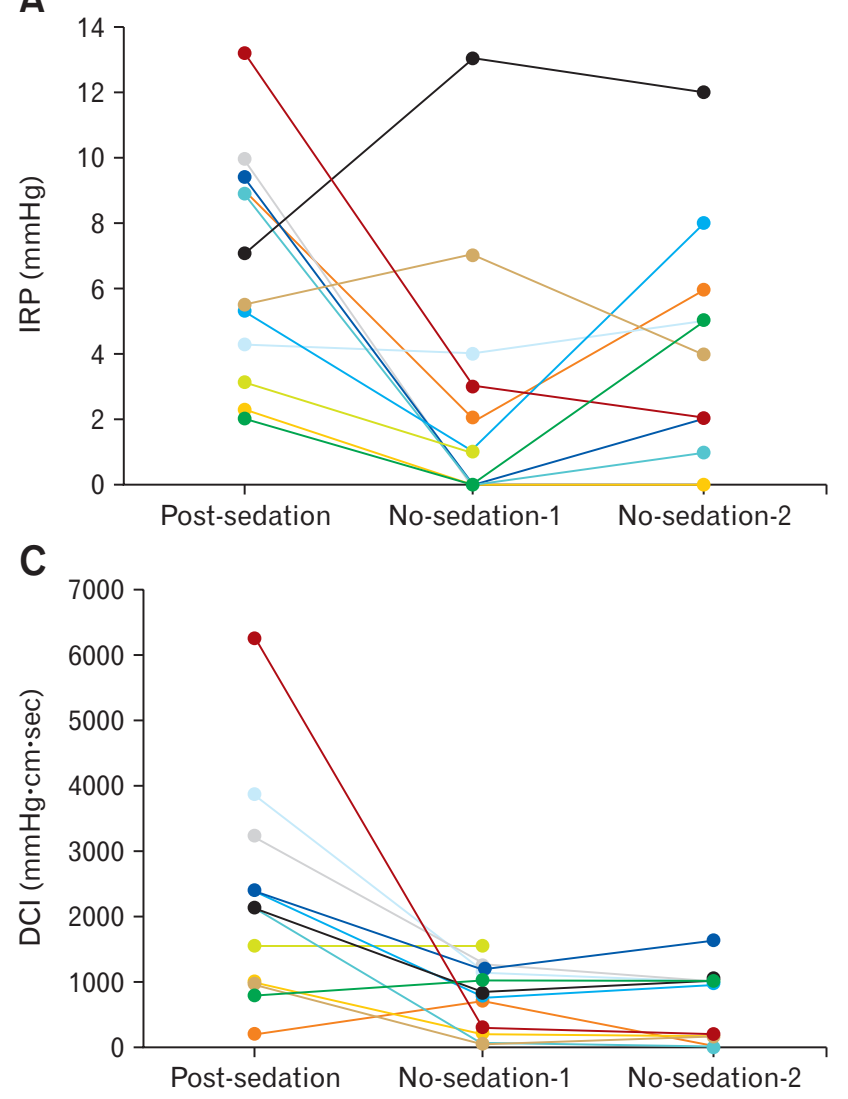

B

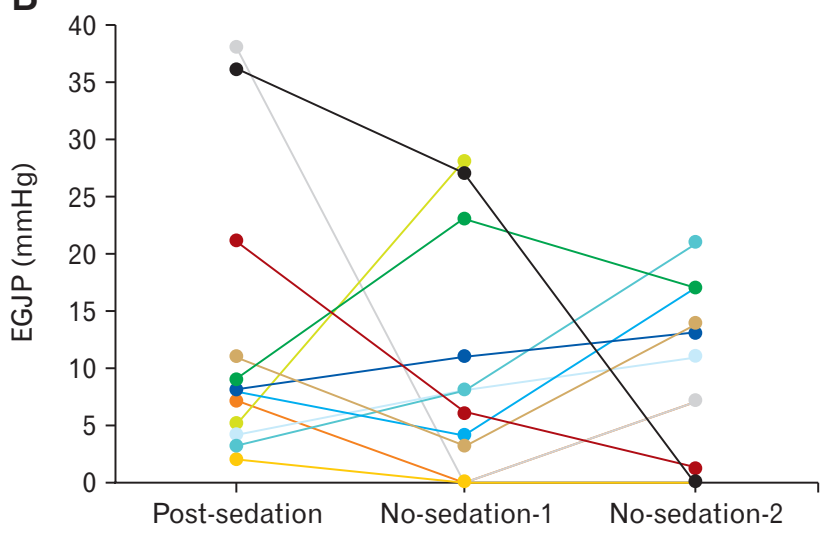

D

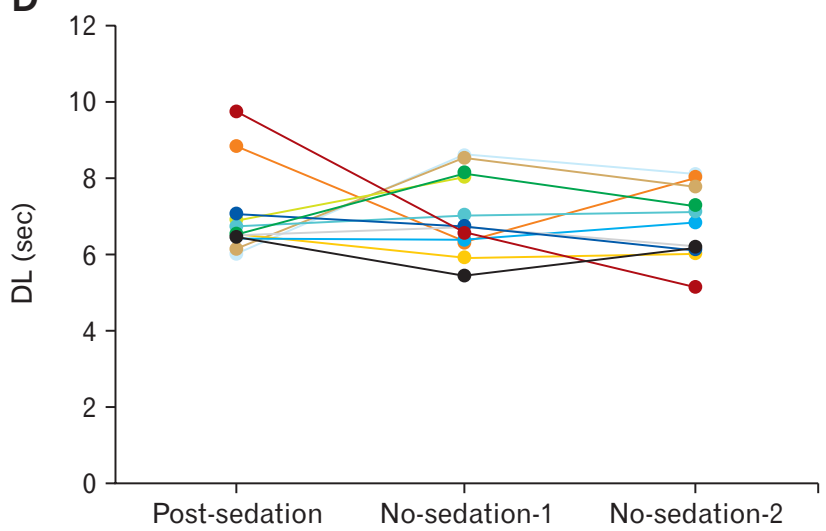

Figure 2. Comparison of upright high-resolution manometry (HRM) metrics of volunteer group. Median integrated relaxation pressure (IRP) (A), esophageal gastric junction pressure (EGJP) (B), distal contractile integral (DCI) (C), and distal latency (DL) (D) were compared across 3 test situations (post-sedation, no-sedation-1, and no-sedation-2) for all of 12 subjects. 
The median (IQR) supine IRP was $10.9(8.2,12.8) \mathrm{mmHg}$ during post-sedation, $5.8(4.6,8.9) \mathrm{mmHg}$ during no-sedation-1, and 7.5 (5.7, 10.5) mmHg during no-sedation-2, $P=0.007$. The post-sedation median IRP was greater than at no-sedation-1 $(P=$ $0.002)$ and trended toward greater than at no-sedation-2 $(P=$ 0.052). Median supine IRP was similar between no-sedation-1 and no-sedation-2, $P=0.201$.

The median (IQR) supine basal esophagogastric junction (EGJ) pressure was $12.5(7.5,15.8) \mathrm{mmHg}$ during post-sedation, $9.0(2.3,15.3) \mathrm{mmHg}$ during no-sedation-1, and $11.0(4.0,15.0)$ mmHg during no-sedation-2, $P=0.528$.

The median (IQR) supine DCI was $2920(1509,4497)$ $\mathrm{mmHg} \cdot \mathrm{cm} \cdot \mathrm{sec}$ during post-sedation, 1117 (622, 1897) $\mathrm{mmHg} \cdot \mathrm{cm} \cdot \mathrm{sec}$ during no-sedation-1, and 1052 (539, 2461) $\mathrm{mmHg} \cdot \mathrm{cm} \cdot \mathrm{sec}$ during no-sedation- $2, P=0.004$. The post-sedation median DCI was greater than both the no-sedation-2 $(P=$ $0.010)$ and the no-sedation-1 $(P=0.011)$.

The median (IQR) supine DL was $6.75(6.51,7.36)$ seconds during post-sedation, $6.90(6.23,8.34)$ seconds during no-seda-

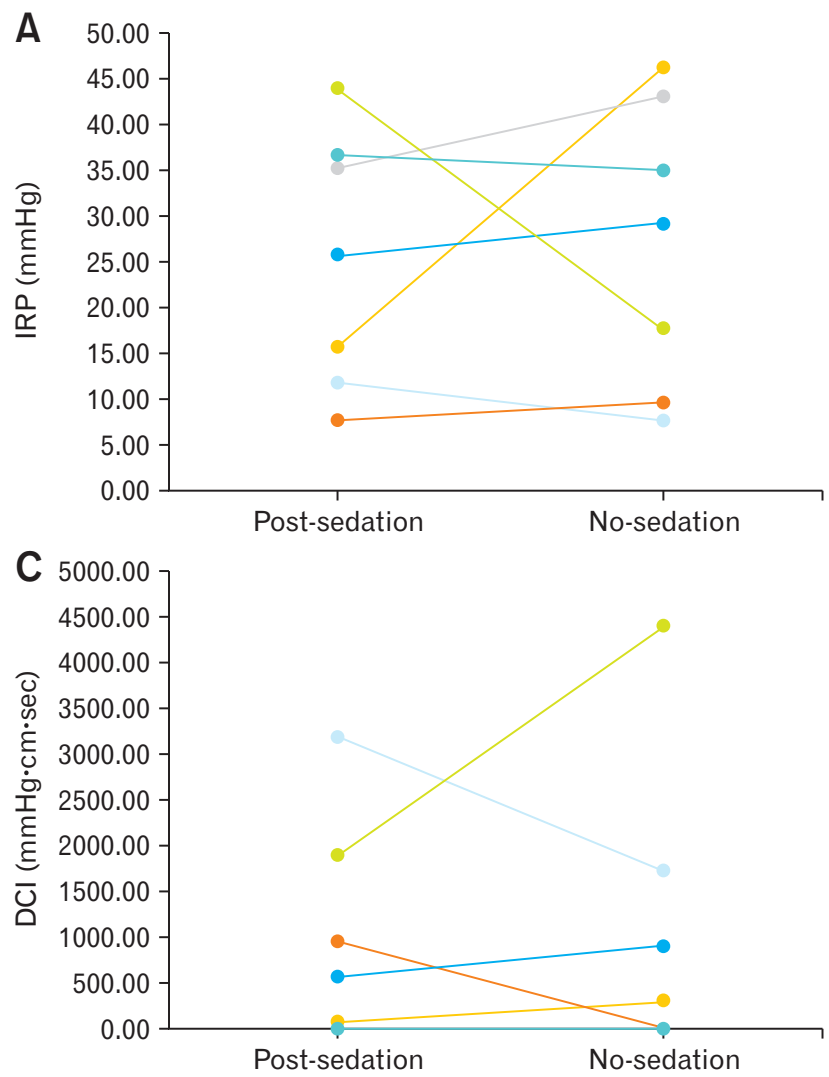

tion-1, $7.05(6.65,7.70)$ seconds during no-sedation-2, $P=0.878$.

The impact of sedation on standard high-resolution manometry metrics in upright in volunteer group (see Fig. 2 and Table)

The median (IQR) upright IRP was $6.3(3.4,9.4) \mathrm{mmHg}$ during post-sedation, $1.0(0.0,3.8) \mathrm{mmHg}$ during no-sedation-1, and $5.0(2.0,6.0) \mathrm{mmHg}$ during no-sedation-2, $P=0.076$.

The median (IQR) upright basal EGJ pressure was 8.0 (4.3, 18.5) $\mathrm{mmHg}$ during post-sedation, $7.0(0.8,20.0) \mathrm{mmHg}$ during no-sedation-1, and $11.0(1.0,17.0) \mathrm{mmHg}$ during no-sedation-2, $P=0.359$

The median (IQR) upright DCI was 2101 (969, 3020) $\mathrm{mmHg} \cdot \mathrm{cm} \cdot \mathrm{sec}$ during post-sedation, 791 (227, 1178) $\mathrm{mmHg} \cdot \mathrm{cm} \cdot \mathrm{sec}$ during no-sedation-1, and 972 (123, 1010) $\mathrm{mmHg} \cdot \mathrm{cm} \cdot \mathrm{sec}$ during no-sedation-2, $P=0.009$, the post-sedation median DCI was greater than no-sedation-2 $(P=0.012)$, and trended toward greater than the no-sedation-1 $(P=0.076)$.

The median (IQR) upright DL was $6.5(6.4,7.0)$ seconds
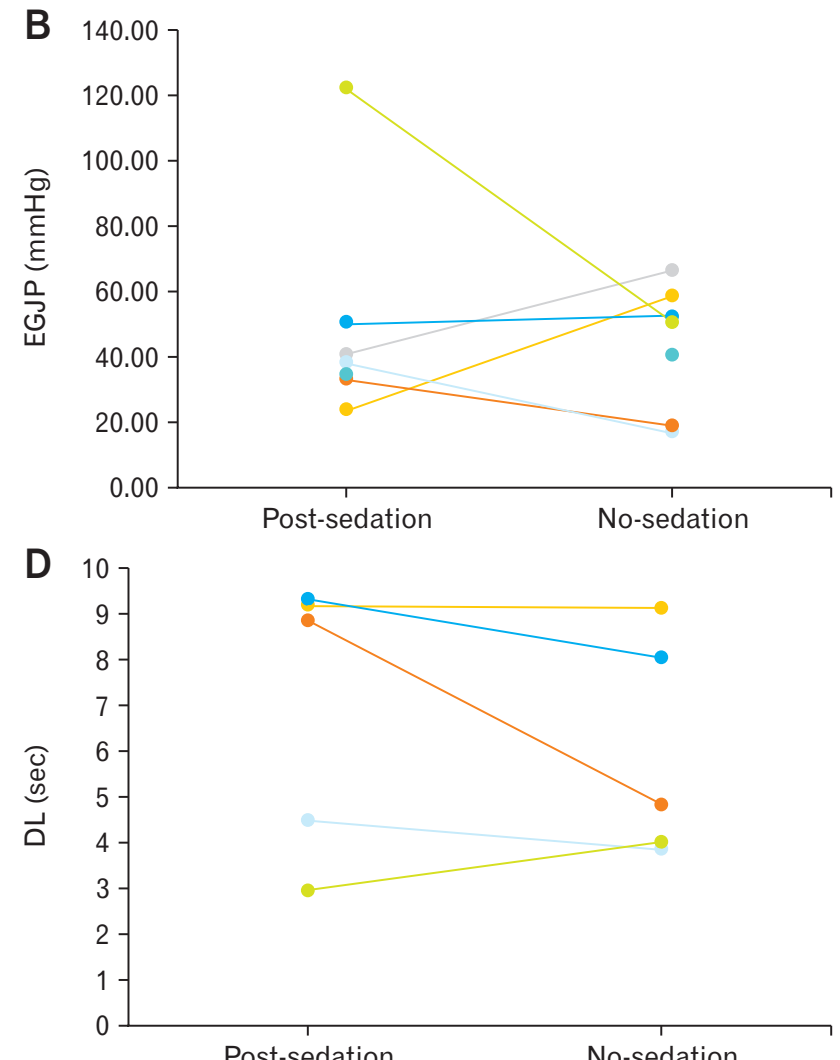

Post-sedation

Figure 3. Comparison of supine high-resolution manometry (HRM) metrics of patient group. Median integrated relaxation pressure (IRP) (A), basal esophageal gastric junction pressure (EGJP) (B), distal contractile integral (DCI) (C), and distal latency (DL) (D) were compared across 2 test situations (post-sedation, no-sedation) for all of 7 patients. 

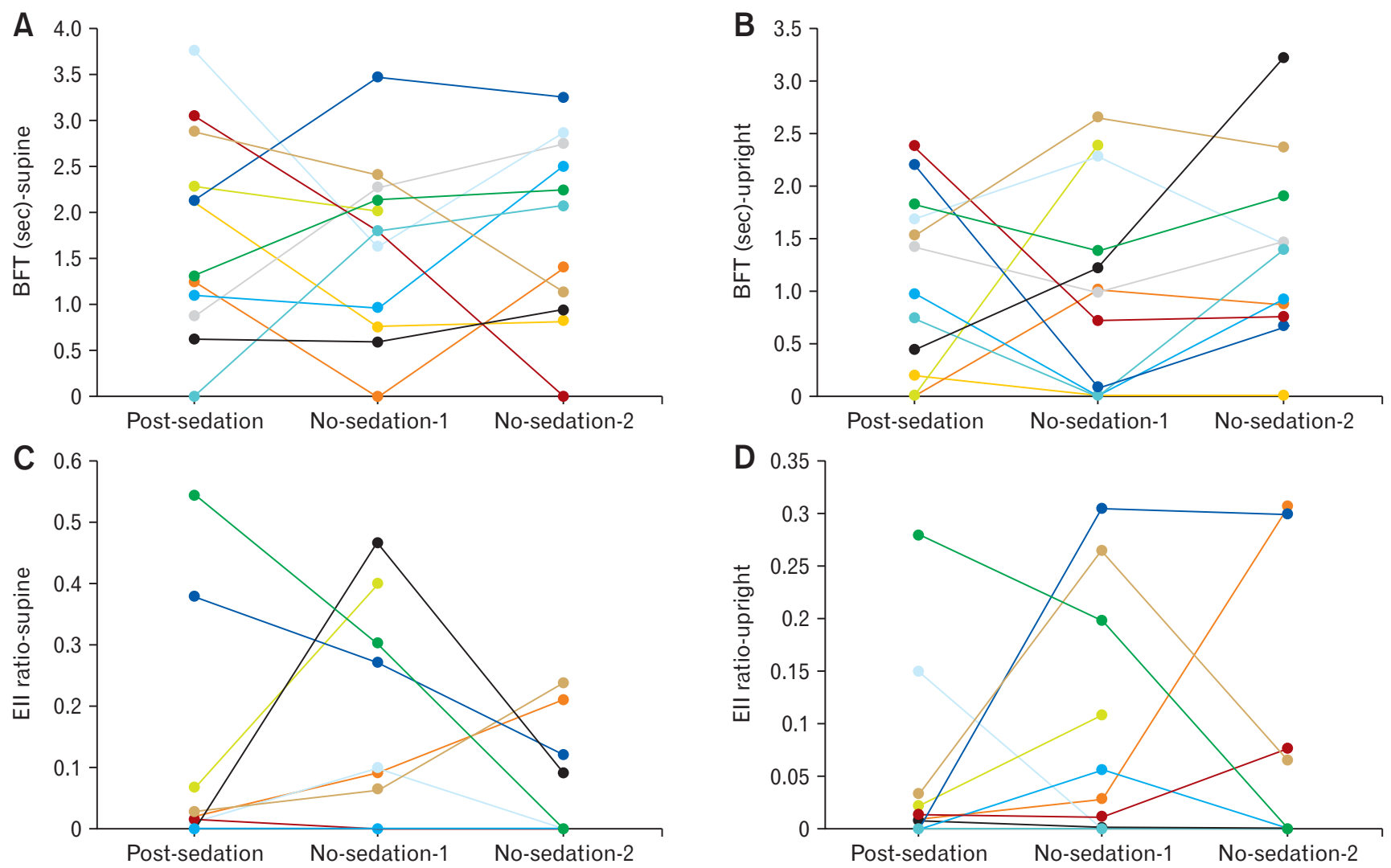

Figure 4. Comparison of supine and upright high-resolution impedance manometry (HRIM) metrics of volunteer group. Median bolus flow time (BFT) and esophageal impedance integral (EII) ratio were compared across 3 test situations (post-sedation, no-sedation-1, and no-sedation-2) for all of subjects.

during post-sedation, 6.7 (6.3, 8.1) seconds during no-sedation-1, $6.8(6.1,7.8)$ seconds during no-sedation-2, $P=0.933$.

The impact of sedation on standard high-resolution manometry metrics in supine in patient group (see Fig. 3)

The median (IQR) supine IRP was $25.80(11.80,36.60)$ $\mathrm{mmHg}$ during post-sedation, $29.05(9.65,42.90) \mathrm{mmHg}$ during no-sedation, $P=0.804$.

The median (IQR) supine basal EGJ pressure was 37.80 $(32.80,50.20) \mathrm{mmHg}$ during post-sedation, $50.00(18.10,58.10)$ mmHg during no-sedation, $P=1.000$.

The Impact of Sedation on High-resolution Impedance Manometry Metrics for Volunteer Group (see Fig. 4)

The impact of sedation on high-resolution impedance manometry metrics in supine (see Fig. 4A and 4C)

The median (IQR) supine BFT was $1.70(0.92,2.73)$ seconds during post-sedation, $1.79(0.81,2.24)$ seconds during no-sedation-1, $2.08(0.94,2.75)$ seconds during no-sedation-2, $P=0.919$

The median (IQR) supine EII ratio was $0.01(0.00,0.06)$ during post-sedation, $0.08(0.00,0.29)$ during no-sedation- $1,0.00$ $(0.00,0.12)$ during no-sedation- $2, P=0.798$.

The impact of sedation on high-resolution impedance manometry metrics in upright (see Fig. 4B and 4D)

The median (IQR) upright BFT was $1.20(0.25,1.79)$ seconds during post-sedation, $1.00(0.02,2.06)$ seconds during nosedation-1, $1.42(0.76,1.92)$ seconds during no-sedation-2, $P=$ 0.693 .

The median (IQR) upright EII ratio was $0.01(0.00,0.03)$ during post-sedation, $0.02(0.00,0.17)$ during no-sedation- $1,0.00$ $(0.00,0.08)$ during no-sedation- $2, P=0.792$.

\section{Discussion}

We evaluated the potential impact of placing an HRIM 
catheter during conscious sedation by comparing HRIM studies performed following sedated upper endoscopy with HRIM studies performed without sedation on asymptomatic volunteers and patients. Our results support that esophageal motility diagnoses remained consistent between post sedated and unsedated studies and that there were small but significant changes in the IRP and DCI for the volunteer group, but no significant differences for the patient group. This suggests that placing an HRIM catheter in a sedated patient remains a viable option for patients that are unable to tolerate awake transnasal HRIM catheter placement.

Studies support that trans-nasal catheter placement fails in $6 \%$ to $19 \%$ of patients and this is primarily related to discomfort and abnormal anatomy. ${ }^{3,4,16}$ Thus, endoscopic placement under conscious sedation has been used to improve comfort of placement and help place the catheter through the EGJ in patients with achalasia and large hernias. However, 2 factors related to this practice could potentially result in differences in manometry metrics and diagnosis: the impact of sedation and the impact of thermal pressure drift.

The effects of opioids on esophageal motor function has gained attention based on several observational studies describing an association of chronic opioid use with esophageal motor disorders, particularly spastic (type III) achalasia and EGJ outflow obstruction. $^{5-8}$ The effects on esophageal motor function of acute opioid or benzodiazepine exposure were also previously investigated. Intravenous morphine yielded increased basal EGJ pressure and increased lower esophageal sphincter (LES) relaxation pressures in 2 studies of healthy controls; contractile amplitudes were unchanged. ${ }^{17,18}$ Intravenous remifentanil was reported to increase LES relaxation pressures (IRP) and reduce distal latencies in healthy volunteers in 1 study, ${ }^{19}$ while another study reported no associated change in IRP, but a reduction in distal latency measures. ${ }^{20}$ This latter study also demonstrated a reduction in trans-EGJ bolus flow utilizing the BFT after remifentanil. ${ }^{20}$ Studies assessing the effect of intravenous midazolam on esophageal motility have yielded inconsistent results: one demonstrated increased basal EGJ pressure and increased LES relaxation pressures among healthy asymptomatic adults, ${ }^{9}$ while another demonstrated no changes in LES pressures among pediatric patients. ${ }^{10}$ Overall, while previous studies reported changes in esophageal motility parameters with administration of intravenous opioids or benzodiazepine generally among healthy volunteers, there were some inconsistencies in findings between studies and all of the studies were reported using experimental conditions.

Another factor that could affect the HRIM findings when performing the HRIM study an hour after placing the catheter is thermal pressure drift. Modest increases in pressure output with the solid-state HRIM assembly utilized in this study may occur over the duration of the thermal and pressure exposure during intraesophageal assembly presence. ${ }^{11,21}$ As the differences between the post-sedation and no-sedation-2 condition in supine IRP and DCI were less pronounced than the comparison with no-sedation-1, the effect of thermal drift may account for the modest changes observed as much if not more so than the effects of the sedative agents (Fig. 1).

In the present study, we investigated the potential impact of a real-world, clinical practice scenario involving performing HRIM after the sedative effects of conscious sedation including both fentanyl and midazolam had waned, eg, approximately 1 hour after upper endoscopy with sedation. In doing so, we observed similarities to the majority of previous similar studies by finding a numeric increase in the IRP at the post-sedation assessment as compared with the no-sedation setting. Differences we observed as compared with previous studies were the lack of change in distal latency or BFT, and the numeric increase in DCI (peristaltic vigor) observed in the post-sedation condition in the volunteer group. While no significant differences of HRM metrics were observed between post sedation and no sedation in the small patients group.

A potential explanation for the differences we observed was that the half-lives of midazolam and fentanyl are 1.5-2.5 hours and 20 minutes, respectively, thus our real-world post-sedation manometry practice may reflect a greater impact of persistent midazolam presence than fentanyl. A strength of the present study was also performing 2 study protocols separated in time to account for the potential impact of thermal pressure drift, in which we did observe even smaller numeric trends toward differences at the no-sedation-2 test scenario in which the thermal pressure drift effect would be more similar to the post-sedation HRIM scenario. Although we did observe the similarly described changes in the various parameters of esophageal motor function with HRIM, when we applied the clinical classification scheme of esophageal motility (the CC) to our study, we demonstrated that the potential clinical impact of these normal-range to normal-range parameter changes would be minimal.

The differences in clinical motility diagnoses observed in our studies included 2 volunteer subjects and 1 patient that had a classification of normal motility in the post-sedation setting, but the 2 volunteer subjects had a classification of IEM in 1 of 2 of the nosedation tests (with normal motility in the other), the patient had IEM in the no-sedation test. The clinical relevance of the IEM diagnosis within the $\mathrm{CC}$ v3.0 remains one of uncertainty, but is a known pattern that can be observed among healthy, asymptomatic subjects. $^{13,22}$ The differences observed between the 2 no-sedation 
scenarios in these volunteer subjects also likely reflect a component of the expected reproducibility of HRIM and esophageal motor function in repeat testing. While study in this area is limited, a previous study on the reproducibility of HRM among healthy volunteers also demonstrated moderate reproducibility in HRM parameters and peristalsis by performing repeat HRM on different days in asymptomatic volunteers. ${ }^{23}$

While strengths of this study include testing a clinicallyrelevant scenario in HRIM performed after upper endoscopy with conscious sedation including both fentanyl and midazolam, applied to clinical motility diagnosis and the control for effects of thermal pressure drift, there are some limitations of this study. One limitation that spans across the body of previous studies on the effects of acute impact of opioids and benzodiazepines on esophageal motility is that only healthy volunteers and a small cohort of esophageal patients were studied. Willingness of patients to repeat HRM is generally limited in the absence of significant clinical changes and most patients that have HRM placed at the time of endoscopy do so for a reason. Although small in sample size, our patient cohort did include both major and minor CC v3.0 classifications, EGJOO, achalasia, distal esophageal spasm, IEM. Thus, while the effects of these pharmacologic stimuli may differ among esophageal patient populations with different esophageal neuronal compositions, eg, loss of inhibitory ganglion in achalasia, we did not observe this finding among our patients. We did observe mild and non-clinically significant increases in IRP and DCI among the healthy controls related to the post-endoscopy test period, but it is conceivable that similar type changes in a patient could push some of the values across a 'threshold' of the classification scheme. ${ }^{13}$ However, it should be recognized that borderline HRM parameters or equivocal diagnoses (eg, EGJ outflow obstruction) in most esophageal motility testing scenarios need to be interpreted in the overall clinical context and may require adjuvant testing (eg, barium esophagram) to support diagnoses with major management implications. While future studies remain needed to evaluate the potential impact of sedative agents on esophageal motility among larger cohorts of esophageal patients, the logistics of performing repeat HRMs among patients that do not require endoscopic placement of HRM catheters pose challenges.

In conclusion, performing HRIM after endoscopy with conscious sedation, and thus the waning effects of midazolam and fentanyl as well as thermal pressure drift, had minimal clinical impact on the motility diagnosis or parameters among asymptomatic volunteers and a small cohort of patients. While further study to assess an impact among patients is warranted, this study suggested that placement of the HRIM catheter while a patient is under sedation may provide an adequate solution in cases that awake HRM catheter placement is not possible.

Financial support: This work was supported by the Public Health service (Grant No. P01 DK117824 [JEP]) and the American College of Gastroenterology (Junior Faculty Development Award [DAC]).

Conflicts of interest: Dustin A Carlson: Medtronic (Speaking and Consulting); Wenjun Kou: Crospon, Inc (consulting); John E Pandolfino: Crospon, Inc (stock options), Given Imaging (Consultant, Grant, and Speaking), Sandhill Scientific (Consulting and Speaking), Takeda (Speaking), Astra Zeneca (Speaking), Medtronic (Speaking and Consulting), Torax (Speaking and Consulting), Ironwood (Consulting), and Impleo (Grant); and $\mathrm{Hui} \mathrm{Su}$, Erica Donnan, Jacqueline Prescott, Alex Decorrevont, Francesca Shilati, and Melina Masihi: none.

Author contributions: Dustin A Carlson and John E Pandolfino designed the study; Dustin A Carlson, Jacqueline Prescott, Alex Decorrevont, Francesca Shilati, Melina Masihi, and Erica Donnan recruited subjects and performed the studies; Hui Su, Dustin A Carlson, Wenjun Kou, and John E Pandolfino performed analysis; and Hui Su, Dustin A Carlson, and John E Pandolfino wrote the paper.

\section{References}

1. Cohen LB, Ladas SD, Vargo JJ, et al. Sedation in digestive endoscopy: the Athens international position statements. Aliment pharmacol Ther 2010;32:425-442.

2. Brun R, Staller K, Viner S, Kuo B. Endoscopically assisted water perfusion esophageal manometry with minimal sedation: technique, indications, and implication on the clinical management. J Clin Gastroenterol 2011;45:759-763.

3. Christian KE, Morris JD, Xie G. Endoscopy- and monitored anesthesia care-assisted high-resolution impedance manometry improves clinical management. Case Rep Gastrointest Med 2018;2018:9720243.

4. Tariq H, Makker J, Chime C, Kamal MU, Rafeeq A, Patel H. Revisiting the reliability of the endoscopy and sedation-assisted high-resolution esophageal motility assessment. Gastroenterology Res 2019;12:157-165.

5. Kraichely RE, Arora AS, Murray JA. Opiate-induced oesophageal dysmotility. Aliment Pharmacol Ther 2010;31:601-606.

6. Ratuapli SK, Crowell MD, DiBaise JK, et al. Opioid-induced esophageal dysfunction (OIED) in patients on chronic opioids. Am J Gastroenterol 2015;110:979-984.

7. Ravi K, Murray JA, Geno DM, Katzka DA. Achalasia and chronic opiate use: innocent bystanders or associated conditions? Dis Esophagus 
2016;29:15-21.

8. Babaei A, Szabo A, Shad S, Massey BT. Chronic daily opioid exposure is associated with dysphagia, esophageal outflow obstruction, and disordered peristalsis. Neurogastroenterol Motil 2019;31:e13601.

9. Marsh JK, Hoffman SM, Dmuchowski CF. Effect of intravenous midazolam on esophageal motility testing in normal human volunteers. Am J Gastroenterol 1993;88:860-863.

10. Fung KP, Math MV, Ho CO, Yap KM. Midazolam as a sedative in esophageal manometry: a study of the effect on esophageal motility. J Pediatr Gastroenterol Nutr 1992;15:85-88.

11. Babaei A, Lin EC, Szabo A, Massey BT. Determinants of pressure drift in manoscan $\left({ }^{\mathrm{TM}}\right)$ esophageal high-resolution manometry system. Neurogastroenterol Motil 2015;27:277-284

12. Carlson DA, Kou W, Lin Z, et al. Normal values of esophageal distensibility and distension-induced contractility measured by functional luminal imaging probe panometry. Clin Gastroenterol Hepatol 2019;17:674-681. e1.

13. Kahrilas PJ, Bredenoord AJ, Fox M, et al. The Chicago classification of esophageal motility disorders, v3.0. Neurogastroenterol Motil 2015;27:160-174.

14. Lin Z, Nicodème F, Lin CY, et al. Parameters for quantifying bolus retention with high-resolution impedance manometry. Neurogastroenterol Motil 2014;26:929-936.

15. Carlson DA, Omari T, Lin Z, et al. High-resolution impedance manometry parameters enhance the esophageal motility evaluation in nonobstructive dysphagia patients without a major Chicago classification motility disorder. Neurogastroenterol Motil 2017;29:e12941.
16. Roman S, Kahrilas PJ, Boris L, Bidari K, Luger D, Pandolfino JE. High-resolution manometry studies are frequently imperfect but usually still interpretable. Clin Gastroenterol Hepatol 2011;9:1050-1055.

17. Dowlatshahi K, Evander A, Walther B, Skinner DB. Influence of morphine on the distal oesophagus and the lower oesophageal sphincter--a manometric study. Gut 1985;26:802-806.

18. Penagini R, Picone A, Bianchi PA. Effect of morphine and naloxone on motor response of the human esophagus to swallowing and distension. Am J Physiol 1996;271(4 Pt 1):G675-G680.

19. Savilampi J, Magnuson A, Ahlstrand R. Effects of remifentanil on esophageal motility: a double-blind, randomized, cross-over study in healthy volunteers. Acta Anaesthesiol Scand 2015;59:1126-1136.

20. Cock C, Doeltgen SH, Omari T, Savilampi J. Effects of remifentanil on esophageal and esophagogastric junction (EGJ) bolus transit in healthy volunteers using novel pressure-flow analysis. Neurogastroenterol Motil Published Online First: 18 Aug 2017. doi: 10.1111/nmo.13191.

21. Robertson EV, Lee YY, Derakhshan MH, et al. High-resolution esophageal manometry: addressing thermal drift of the manoscan system. Neurogastroenterol Motil 2012;24:61-64, e11.

22. Pandolfino JE, Ghosh SK, Rice J, Clarke JO, Kwiatek MA, Kahrilas PJ. Classifying esophageal motility by pressure topography characteristics: a study of 400 patients and 75 controls. Am J Gastroenterol 2008;103:2737.

23. Bogte A, Bredenoord AJ, Oors J, Siersema PD, Smout AJ. Reproducibility of esophageal high-resolution manometry. Neurogastroenterol Motil 2011;23:e271-e276. 